\title{
Electrochemical Oxidative Fluorination of an Oxide Perovskite
}

Nicholas H. Bashian, ${ }^{\dagger}$ Mateusz Zuba, ${ }^{\ddagger}$ Ahamed Irshad, ${ }^{\dagger}$ Shona M. Becwar, ${ }^{\llbracket}$ Julija Vinckeviciute, ${ }^{\S}$ Warda Rahim,,$\perp$ Kent J. Griffith, $\#$ Eric T. McClure, ${ }^{\dagger}$ Joseph K. Papp, ${ }^{@}$ Bryan

D. McCloskey, ${ }^{\circledR}$ David O. Scanlon, $\|, \perp, \triangle, \nabla$ Bradley F. Chmelka, ${ }^{\llbracket}$ Anton Van der Ven, ${ }^{\S}$ S. R. Narayanan, ${ }^{\dagger}$ Louis F. J. Piper, ${ }^{*,+, \dagger \dagger}$ and Brent C. Melot ${ }^{*, \dagger}$

$\dagger$ Department of Chemistry, University of Southern California, Los Angeles, CA 90089, United States $\ddagger$ Department of Physics, Applied Physics and Astronomy, Binghamton University, Binghamton, NY 13902, United States

\Department of Chemical Engineering, University of California, Santa Barbara, Santa Barbara, CA 93106, United States

$\S$ Materials Department, University of California, Santa Barbara, Santa Barbara, CA 93106, United States \|Thomas Young Centre, University College London, Gower Street, London, WC1E 6BT, United Kingdom $\perp$ Department of Chemistry, University College London, 20 Gordon Street, London, WC1H 0AJ, United Kingdom \#Department of Chemistry, University of Cambridge, Lensfield Road, Cambridge CB2 1EW, United Kingdom

@ Department of Chemical and Biomolecular Engineering, University of California, Berkeley, CA 94720, United States

$\triangle$ Diamond Light Source Ltd., Diamond House, Harwell Science and Innovation Campus, Didcot, Oxfordshire OX11 ODE, United Kingdom

$\nabla$ The Faraday Institution, Quad One, Harwell Science and Innovation Campus, Didcot, United Kingdom †† Materials Science 83 Engineering, Binghamton University, Binghamton, NY 13902, United States

E-mail: Ipiper@binghamton.edu; melot@usc.edu 


\begin{abstract}
We report the successful electrochemical intercalation of F-ions into a densely packed perovskite oxide from a liquid electrolyte at room temperature. Using galvanostatic oxidation and electrochemical impedance spectroscopy coupled with operando X-ray diffraction, we show that roughly 0.5 equivalents of F-ions can be inserted onto the vacant $\mathrm{A}$-site of the perovskite $\mathrm{ReO}_{3}$. Density functional theory calculations indicate that the intercalated phase is thermodynamically unfavorable compared to other less densely packed polymorphs of $\mathrm{ReO}_{3} \mathrm{~F}$. Pairing X-ray spectroscopy, neutron total scattering measurements, and magic-angle spinning ${ }^{19} \mathrm{~F}$ NMR confirms a rapid decomposition of the product on removal from the cell but nevertheless, these results clearly demonstrate that small anions like fluoride can be intercalated into solids as readily as alkali cations at room temperature, which opens new opportunities to electrochemically fluorinate many new materials.
\end{abstract}

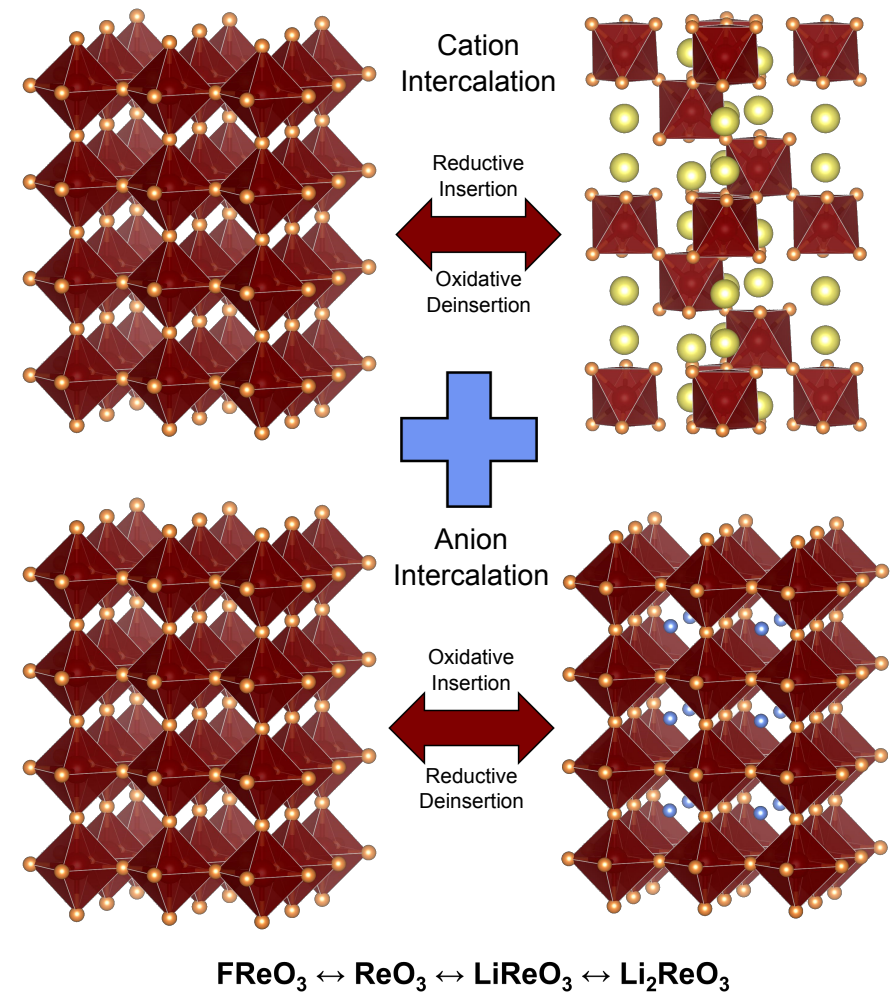

Scheme 1: Combining anion and cation insertion processes could break gravimetric capacity limits by allowing for multi-electron redox processes. 
The ability to topotactically control the insertion of ions into crystalline hosts is critical to a range of technologies, including energy storage, electrochromic displays, superconductivity, and catalysis. ${ }^{1-3}$ While research on cationic intercalation chemistry has flourished, ${ }^{4}$ anionic intercalation has largely been overlooked ${ }^{5}$ with most work in the area focused on bulky polyanionic groups inserted into layered materials. ${ }^{6}$ Given their substantially larger radius and negative charge polarity, anions require drastically different conditions from cations to promote fast ionic mobility. ${ }^{7,8}$ The earliest work on anion insertion chemistry was performed by Schafhaeutl and then built upon by Rüdorff and Hofmann when they showed that $\mathrm{SO}_{4}^{2-}$ could intercalate between the sheets of graphite in the presence of a chemical oxidant. ${ }^{9,10}$ Electrochemical intercalation into crystalline graphite was not achieved until the early 1980's using slow cyclic voltammetry to drive the ionic diffusion of species such as $\mathrm{ClO}_{4}^{-}, \mathrm{SO}_{4}^{2-}$, and $\mathrm{BF}_{4}^{-} \cdot{ }^{11,12}$ Mallouk and Bartlett later described the chemical insertion of fluoride in graphite using HF, identifying the formation of biflouride $\mathrm{C}_{12} \mathrm{HF}_{2}{ }^{13}$ Carlin then built on this work to develop a symmetric graphite cell that intercalated bulky ions like imidazolium at the cathode and $\mathrm{AlCl}_{4}^{-}$at the anode to create one of the first embodiments of a dual-ion battery. ${ }^{14}$

While the intercalation chemistry of bulky polyanionic groups have received some attention, ${ }^{15,16}$ the electrochemistry of smaller species like fluoride has been mostly limited to conversion-based systems where a reversible chemical transformation is used. ${ }^{17-19}$ A critical challenge facing these systems is that they rely almost exclusively on solidstate fluoride conductors, like Ba-doped $\mathrm{LaF}_{3},{ }^{20-22}$ sandwiched between metal and metal-fluoride electrodes. ${ }^{23,24}$ Fichtner and coworkers, for example, have demonstrated a F-ion battery consisting of a $\mathrm{CuF}_{2}$ cathode cycled against a film of La metal which results in the reduction of the cathode to $\mathrm{Cu}$ metal and a conversion to $\mathrm{LaF}_{3}$ on the anode side. ${ }^{25}$ More recently, Clemens and coworkers have leveraged all solid-state cells, showing some success with intercalating fluoride into $\mathrm{La}_{2} \mathrm{CoO}_{4}$, though the poor conductivity of the $\mathrm{LaF}_{3}$ solid electrolyte required operating cells at $170{ }^{\circ} \mathrm{C} .{ }^{26,27}$ This was very recently followed by the work of Banerjee et al. who demonstrated chemical insertion and removal of fluoride from $\mathrm{FeSb}_{2} \mathrm{O}_{4}$ at room temperature using chemical redox methods. ${ }^{28}$

The high operating temperature of these solids precludes their use in any practical devices, which would require a fluoride electrolyte with high mobility at room-temperature. Christe and others reported one of the earliest advances towards such an electrolyte when they reported that quaternary ammonium salts can stabilize free fluoride in THF. ${ }^{29,30}$ More recently, Davis et al. showed that salts of N,N,N-trimethyl-N-neopentylammonium fluoride $(\mathrm{Np} 1 \mathrm{~F})$ dissolved in fluoroether solvents could facilitate the stable cycling of $\mathrm{CuF}_{2} @ \mathrm{LaF}_{3}$ core-shell nanoparticles as conversion cathodes. ${ }^{31}$ This seminal work represented some of the first tangible evidence that electrochemical energy storage can leverage anions like fluoride in similar ways to lithium.

Drawing inspiration from this extensive work in the literature, we sought to explore the fundamental structural requirements that facilitate fluoride ion mobility in the solid state. Our recent work with $\mathrm{ReO}_{3}$, in which we studied the fundamental structural distortions that occur during lithiation, ${ }^{32}$ offered a natural starting material for these studies given that the A-site vacancy within perovskite provides an obvious interstitial where the fluoride could 
intercalate. Demonstrated in Scheme 1, dual cation and anion insertion could provide an exciting breakthrough to increase capacity limits. Furthermore, $\mathrm{ReO}_{3}$ is intrinsically metallic, which removes the need for carbon coating and drastically simplifies the interpretation of spectroscopic data.

In this contribution, we demonstrate the first observation of oxidative intercalation of fluoride into an oxide host from a liquid fluoride electrolyte at room temperature. We find that the negative charge and larger ionic radii of $\mathrm{F}^{-}$demand a vastly different mechanism for intercalation compared to what was observed during Li insertion. Using complementary spectroscopic and structural tools, combined with operando electrochemical characterization, we show that fluoride-ions incorporate onto the A-site of $\mathrm{ReO}_{3}$ during cycling, which creates a highly unstable phase that rapidly decomposes. While this decomposition prevents reversible cycling, it also provides insight into a completely new mechanism for fluoride intercalation under relatively mild and tunable electrochemical conditions and provides insight into how changing the host composition could stabilize F-ions on the A-site.

$\mathrm{ReO}_{3}$ was chosen as a model electrode compound to investigate the fundamentals of fluoride intercalation for several reasons, including the high degree of covalency in bonding, the existence of vacancies to accommodate ions, and its inherent metallic character. $\mathrm{ReO}_{3}$ crystallizes in the $P m \overline{3} m$ space group (\#221) and possesses a perovskitelike structure composed of highly symmetric corner sharing $\mathrm{ReO}_{6}$ octahedra in a perfectly cubic arrangement with an empty $A$-site that creates large three dimensional channels for ionic diffusion ${ }^{33}$ as shown in Figure 1(a). While cation insertion has been demonstrated previously, the close-packed oxygen network [Figure 1(b)] makes anion transport pathways less obvious.

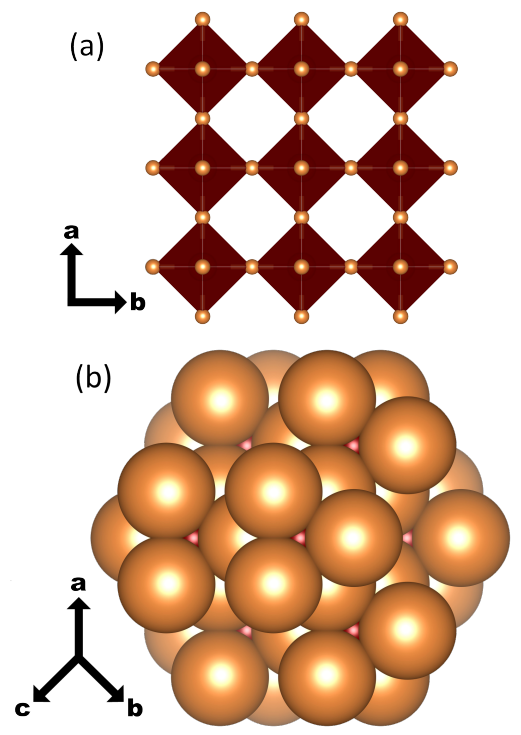

Figure 1: (a) The structure of $\mathrm{ReO}_{3}$ with orange oxygen spheres and maroon rhenium-centered octahedra, shown along [001]. (b) The structure or $\mathrm{ReO}_{3}$ is displayed along the [111] using the ionic radii of $\mathrm{Re}$ and $\mathrm{O}$, emphasizing the tight packing of anions.

The synthesis of $\mathrm{ReO}_{3}$, described in the Experimental Methods section of the Supporting Information, yields highly uniform and crystalline nanoparticles between 20 and 50 nanometers in diameter. $\mathrm{ReO}_{3}$ is one of the few 
metallic oxides, exhibiting a conductivity on the order of $10^{-5} \mathrm{~S} / \mathrm{cm},{ }^{34}$ making it an excellent model battery electrode because it is possible to prepare test cells without conductive carbon additives, which would complicate many spectroscopic measurements. Additionally, using test electrodes comprised entirely of $\mathrm{ReO}_{3}$ removes any potential side reactions related to carbon or binder additives, an important consideration in new cell design.

In an attempt to insert fluoride into $\mathrm{ReO}_{3}, \mathrm{~F}$-ion half cells were assembled using a $\mathrm{Cu}$ metal combined counter and reference electrode, and an organic liquid fluoride electrolyte, as described in the S.I. Experimental Methods section. Glass fiber separators soaked in an electrolyte of tetra-n-butylammonium fluoride (TBAF) dissolved in tetrahydrofuran (THF) served as the source of free fluoride ions between the working and counter electrode. TBAF was chosen for its relative stability and wide electrochemical window as well as a reasonable fluoride-ion conductivity. As shown in Figure 2(a), the oxidation reaction produces a smooth voltage curve with an initial plateau region followed by a smooth voltage rise until approximately $\mathrm{F}_{0.6} \mathrm{ReO}_{3}$. While the use of $\mathrm{Cu}$ metal provides a consistent reference

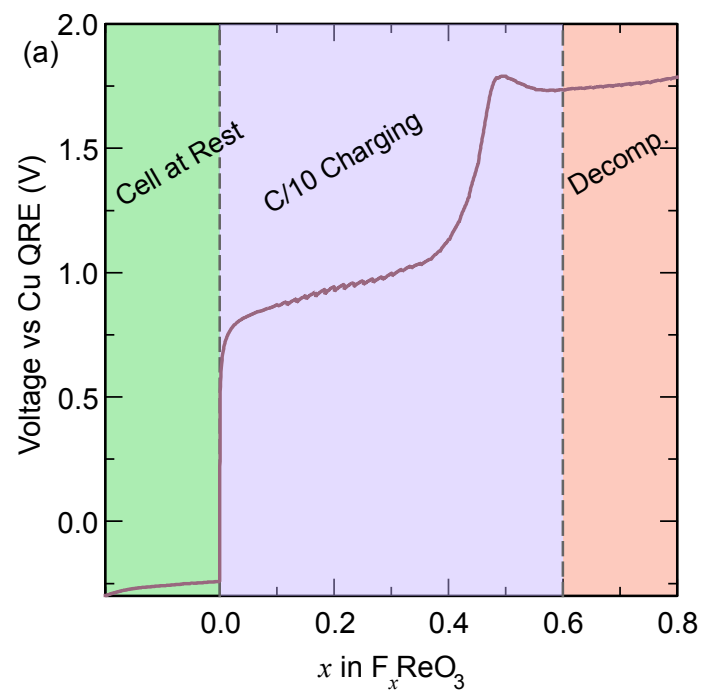

(b)

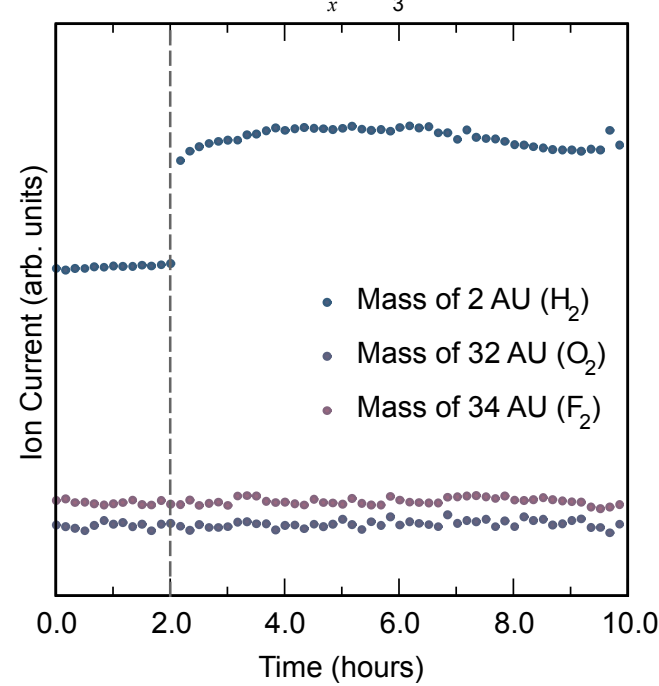

Figure 2: (a): Galvanostatic charge curve of a fluoride-ion half cell during mass spectrometry measurements with a $\mathrm{ReO}_{3}$ working electrode and $\mathrm{Cu}$ metal counter and reference electrode. (b): Differential electrochemical mass spectrometry identified $\mathrm{H}_{2}$ evolution in fluoride ion battery half cells, however no $\mathrm{O}_{2}$ or $\mathrm{F}_{2}$ generation was seen. 
potential, it cannot be further reduced which raised questions about the nature of the counter reaction occurring at the cathode. To better understand this counter reaction, Differential Electrochemical Mass Spectrometry (DEMS) measurements were employed to monitor any evolution of gas in situ during the cycling at both the anode and cathode. Several gases were monitored during oxidation, including $\mathrm{O}_{2}, \mathrm{~F}_{2}$, and $\mathrm{CO}_{2}$ (shown in S.I. Figure S8), but as seen in Figure 2(b), the only gas that was observed to evolve corresponded to $\mathrm{H}_{2}$, which persisted throughout charging. Therefore, the reductive counter reaction is found to be associated with a hydrogen evolution reaction (HER) from an attack of the THF or TBAF salt. The lack of oxygen release from $\mathrm{ReO}_{3}$ throughout the measurement is notable given that the quasi-reference potentials observed scale to be far in excess of $4 \mathrm{~V} \mathrm{vs.} \mathrm{Li}^{+} / \mathrm{Li}$. This suggests there is unlikely to be very much decomposition of the $\mathrm{ReO}_{3}$ particles through the release of lattice oxygen in the manner previously reported for other metal oxides. ${ }^{35,36}$ This high voltage stability is most likely associated with the covalent character of the material - the strong hybridization between the metal and oxygen orbitals make it very difficult to strip oxygen out of the lattice.

To validate the liquid-phase electrochemistry, all-solid state cells were prepared using a Ba-doped $\mathrm{LaF}_{3}$ electrolyte to eliminate solvent decomposition and only allow for redox reactions involving the transport of fluoride-ions. Given the slow fluoride conductivity of the solid electrolyte, it was necessary to heat the cells to $150{ }^{\circ} \mathrm{C}$ during cycling in order to facilitate facile ion transport in the solid electrolyte. As the $\mathrm{H}_{2}$ evolution reaction observed in the liquid electrolyte half cells in Figure 2(b) is not possible in the solid state, a $\mathrm{CuF}_{2}$ counter electrode was chosen as a reductive fluoride source. A similar voltage profile was observed during charging of the solid state cells as that of the liquid cells, however, the sluggish nature of F-ion transport in the solid state led to a high cell resistance in the solid state cells which were tested. Therefore Galvanostatic Intermittent Titration (GITT) measurements were used to examine the equilibrium voltage of the reaction. GITT measurements were also performed on cells with a liquid electrolyte allowing for direct comparison to the electrochemistry performed in solid state cells. As shown in Figure 3, a similar voltage relaxation profile was observed in both solid state and liquid cells, suggesting a very similar oxidative reaction at the $\mathrm{ReO}_{3}$ electrode for both cell designs.

Electrochemical Impedance Spectroscopy (EIS) studies on the solid electrolyte cell were also used to monitor variations in the charge transfer and mass transfer resistances at different levels of oxidation. The Nyquist plot in S.I. Figure S7(a) shows a depressed semicircle in the high frequency region characteristic of charge transfer resistance $\left(\mathrm{R}_{c t}\right)$ in parallel with the interfacial double layer capacitance $\left(\mathrm{C}_{d l}\right)$. The values of $\mathrm{R}_{c t}$ do not change significantly until $x=0.5$ and a small rise of $2-3 \Omega$ is noticed thereafter. The increase in $\mathrm{R}_{c t}$ occurs concurrently with the transition from the initial sloping voltage to a higher voltage plateau. In the low-frequency region of the impedance spectrum, the impedance rises steadily with decreasing frequency with a phase angle of approximately $45^{\circ}$ suggesting a diffusion-limited process. Considering the diffusion length and the thickness of the sample, this section of the impedance spectrum can be modeled with the semi-infinite linear diffusion boundary conditions, as described in the S.I. Impedance Spectroscopy section. A decrease in $D$ is observed with increasing state of charge, 


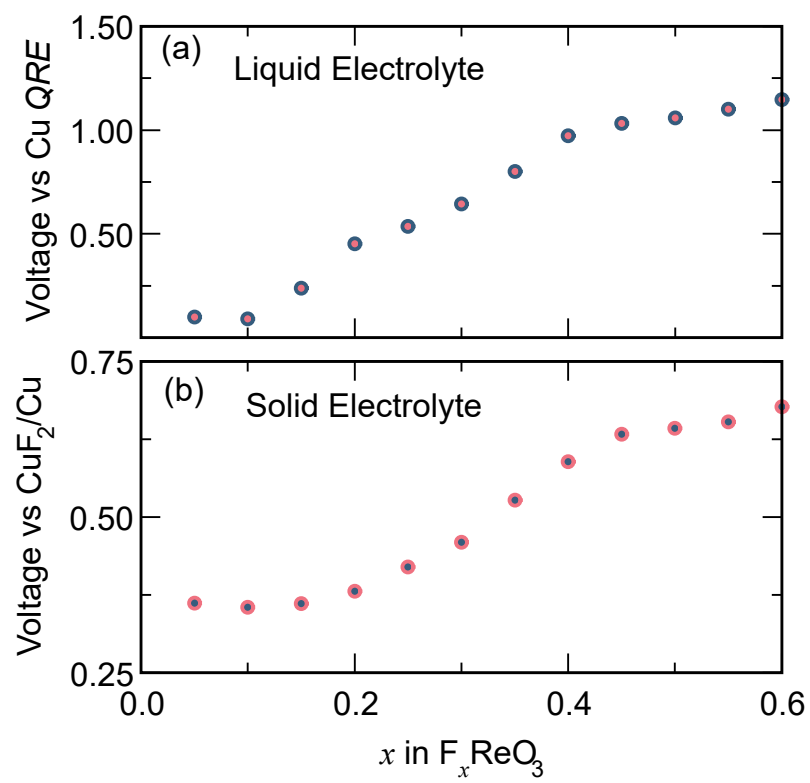

Figure 3: Galvanostatic intermittent titration measurements in (a) liquid electrolyte cells prepared with TBAF electrolyte show similar open circuit voltage traces when compared to (b) voltage traces from all solid state cells.

as shown in S.I. Table T1, suggesting that changes to the material during charging hinder fluoride transport.

Having validated that the oxidative currents were indeed associated with a reaction at the $\mathrm{ReO}_{3}$ electrode, and were not simply electrolyte decomposition, we turned to developing a mechanistic understanding of how fluoride intercalation could occur within the structure of $\mathrm{ReO}_{3}$. Operando X-ray Diffraction (XRD) patterns were collected during oxidation until an equivalent current for one fluoride per formula unit had been passed. As seen in Figures 4(a) and 4(b), the (111) reflection continuously shifts very slightly to higher angles, indicating a small contraction of the unit cell during charging but no new peaks evolve nor do any of the existing peaks split to suggest changes in the cubic symmetry of the lattice. Beyond a nominal fluoride content of roughly 0.6 , the intensity of diffraction patterns begin to significantly decrease, indicating the onset of decomposition as illustrated in S.I. Figure S5. We note, however, that immediately after exposure to the TBAF electrolyte, and prior to cycling, several low intensity peaks appear in data obtained at the synchrotron (further demonstrated in S.I. Figure S3). These peaks cannot be seen with laboratory X-ray sources and do not change position or intensity at any point during the cycling (see S.I. Figure S4), and are therefore believed to be associated with an electrochemically inert contaminant associated with the electrolyte solution.

While the extremely minor changes seen in the diffraction patterns initially seem puzzling, a careful look at the cubic structure shows that placing fluoride ions onto the vacant A-site would not break any of the symmetry elements within the parent perovskite (see S.I. Figure S6), which is consistent with the absence of any new diffraction peaks. In parallel, ex situ electron microscopy and electron energy loss spectroscopy (EELS) mapping were used to monitor the particle morphology and distribution of fluoride in the electrode after charging. As shown in Figure 4(c), the particles maintain their cubic morphology with signs of crystallinity suggesting that the $\mathrm{ReO}_{3}$ does not dissolve or directly extrude any secondary phases during oxidation. EELS measurements, shown in Figure 4(d) show an uneven fluoride 
distribution throughout the particles with fluoride rich regions near the surfaces. Nevertheless, there is no evidence that the morphology of the $\mathrm{ReO}_{3}$ nanoparticles alters during cycling, which rules out a dissolution-precipitation style reaction. Furthermore the fluoride persists on the particles even after washing the ex situ samples thoroughly to remove any residual electrolyte.

Ex situ X-ray Emission Spectroscopy (XES) was also collected on similarly washed samples to more directly track the fluoride content of the oxidized electrodes. The greater penetration depth allows for sensitivity to bulk fluoride rather than simply probing species isolated to the surface. By comparing the intensity of the spectra from the $\mathrm{O}$ $2 p$ orbitals to the $\mathrm{F} 1 s$ orbitals, relative changes in the bulk fluoride content are clearly seen. As shown in Figure 4(e), the intensity of the peak at approximately $677 \mathrm{eV}$, associated with the $\mathrm{F} 1 \mathrm{~s}$ orbitals from bulk fluoride species, grows with increasing state of charge while that of the $\mathrm{O} 2 p$ peak at $531 \mathrm{eV}$ remains constant. As demonstrated by the DEMS measurements in Figure 2(b), there is no reason to believe that $\mathrm{O}_{2}$ is released from the lattice during oxidation; thus the variation in the $\mathrm{F}$ peak corresponds an increase in the fluoride content throughout the $\mathrm{ReO}_{3}$ electrode during charge. In contrast, the states associated with the lattice oxygen of $\mathrm{ReO}_{3}$ stay relatively constant, but do show some restructuring due to changes in the chemical environment of the materials, which will be discussed in greater detail later. Taking these results together, it seems clear that the fluoride reacts with the $\mathrm{ReO}_{3}$, but it is not immediately clear whether the fluoride intercalates into the structure or if there is a more nuanced transformation that is missed by the operando X-ray diffraction experiments.
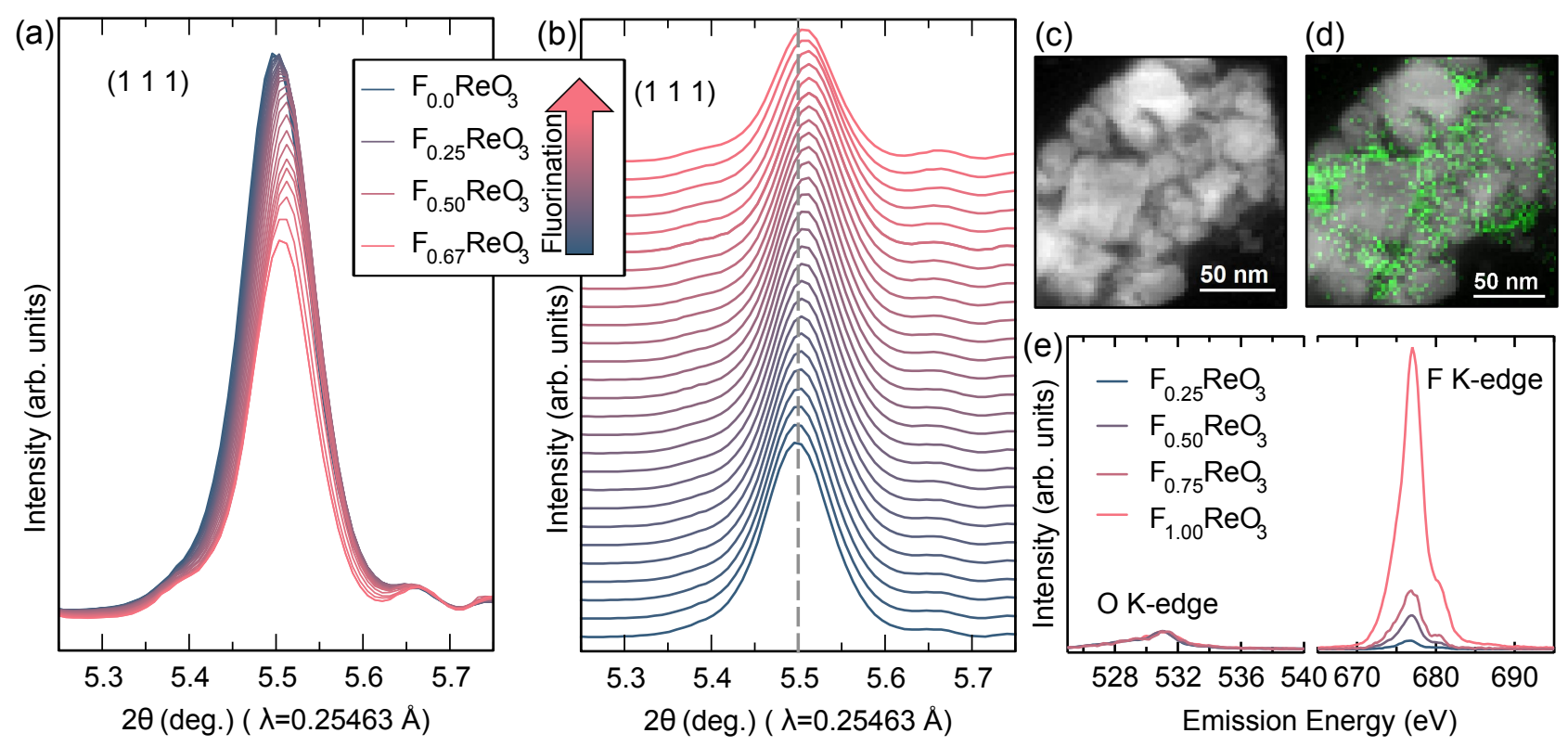

Figure 4: (a): Operando synchrotron X-ray powder diffraction captured at various states of charge during the fluorination of $\mathrm{ReO}_{3}$. A loss of diffracted intensity is observed during charging. (b): The (111) reflection is highlighted to demonstrate the slight shift to higher $2 \theta$ values in conjunction with a loss of diffracted intensity at higher states of charge. (c): TEM images of fluorinated $\mathrm{ReO}_{3}$ show the cubic particle morphology is maintained while (d) EELS spectra show a F-rich shell on the particles. (e): X-ray emission spectroscopy of $\mathrm{F}_{x} \mathrm{ReO}_{3}$ at various states of charge shows an increase in $\mathrm{F}$ signal due to fluoride intercalation.

In order to to assess the thermodynamics of various reaction mechanisms on the insertion of $\mathrm{F}$ into $\mathrm{ReO}_{3}$, first- 
principles density functional theory (DFT) calculations were performed. We first explored topotactic insertion by enumerating different F-vacancy orderings over the A-sites of $\mathrm{ReO}_{3}$. Figure $5(\mathrm{a})$ shows the calculated formation energies of these orderings at a variety of concentrations using the perovskite forms of $\mathrm{ReO}_{3}$ and fully fluorinated $\mathrm{FReO}_{3}$ as the reference states. The formation energies are very slightly negative indicating that F-vacancy ordering over the A sites might be expected at low temperatures, while a solid solution will emerge at elevated temperatures provided the $\mathrm{ReO}_{3}$ perovskite host remains intact.

An alternative to topotactic insertion would be a reconstructive or conversion reaction mechanism, ${ }^{37}$ whereby a completely new crystal structure emerges during oxidation. A survey of the Inorganic Crystal Structure Database ${ }^{38}$ shows the existence of a monoclinic form of $\mathrm{ReO}_{3} \mathrm{~F}$ where the structure consists of edge-sharing chains of octahedrally coordinated $\mathrm{ReO}_{4} \mathrm{~F}_{2}$ units with two fluoride ions sitting along common edges of the polyhedra (see S.I. Figure S13). ${ }^{39}$ In the following, we distinguish the intercalated phase from this one by writing the compositions as $\mathrm{F}_{x} \mathrm{ReO}_{3}$ or mono$\mathrm{ReO}_{3} \mathrm{~F}$ respectively. The energy of this compound is predicted to be more than $2 \mathrm{eV}$ lower per formula unit than that of the fully intercalated perovskite form of $\mathrm{F}_{x} \mathrm{ReO}_{3}$, which points to the existence of an enormous thermodynamic driving force for a reconstructive transformation.

Given that the crystal structure of the mono- $\mathrm{ReO}_{3} \mathrm{~F}$ form is very different from that of the perovskite form of $\mathrm{ReO}_{3}$, it may not be kinetically accessible at room temperature. We therefore decided to explore other hypothetical structures that may be more readily accessed during $\mathrm{F}^{-}$insertion into $\mathrm{ReO}_{3}$. One such structure can be obtained by inserting $\mathrm{F}$ in the vacant $\mathrm{A}$-sites, followed by a coordinated migration of Re cations from their octahedral sites to a newly created tetrahedral interstitial, referred to as $\mathrm{F}_{x} \mathrm{ReO}_{3}-1 \mathrm{~F}$ (see structure in Figure 6). This hopping of the cations was considered for several different compositions of fluoride but was repeatedly found to be higher in energy than when there is no migration of Re out of the octahedral sites. Similarly, geometric relaxations of the structures also indicated that the forces on the atoms can be fully minimized by distorting Re out of the center of the octahedra towards one of the triangular faces of the octahedra to create a highly distorted seven coordinate environment, referred to as seven-coordinate $\mathrm{ReO}_{3} \mathrm{~F}$ (see S.I. Figure S12). This too was found to be far less favorable than F-ions sitting in the empty cages of the cubic form of $\mathrm{ReO}_{3}$.

Interestingly, however, we find that when all of the A-sites are filled by fluoride and all of the Re ions simultaneously migrate into the tetrahedral interstitials, as illustrated in S.I. Figure S15 this structure is significantly lower in energy than the structure with fluoride only on the A-site, considered as intermediate compositions. This phase, referred to as tet- $\mathrm{FReO}_{3}$, is best viewed as a molecular crystal with covalently bonded $\mathrm{ReO}_{3} \mathrm{~F}$ tetrahedra that are loosely held together by weaker van der Waals forces. In this work, we have considered the polar form of the structure where the apical F-ions on each tetrahedron point in the same direction, but, in reality, the tetrahedra are likely to be far more disordered, which we have not considered here at all.

These calculations clearly suggest that if fluoride-intercalated $\mathrm{ReO}_{3}$ were actually to be obtained, there would be a very strong driving force for it to transform into either the mono- $\mathrm{ReO}_{3}$ polymorph reported in the materials project 
or, while not as likely, the form with all of the Re migrated to tetrahedral interstitals [S.I. Figure S15]. At small values of $x$, i.e. less than $\mathrm{F}_{0.5} \mathrm{ReO}_{3}$, the energy to form the phase with Re moved into the newly formed tetrahedral interstitial is actually higher than that of simply placing F-ions on the A-site.

This suggests that a reconstructive reaction could occur through a two-phase mechanism, and, indeed, an application of the common tangent construction to all the formation energies of structures that are kinetically accessible predicts a two-phase coexistence between $\mathrm{ReO}_{3}$ and tet- $\mathrm{FReO}_{3}$ as shown by the dashed gray line in Figure 5(a). This partitioning into two-phases could be expected to result in a core-shell geometry, in which the new tet-FReO 3 phase forms on the surface and grows inward, consuming the original $\mathrm{ReO}_{3}$ core, which appears consistent with the EELS maps but, in principle, should have been observable in the operando diffraction patterns if the shell were fully crystalline. However, given that the mono- $\mathrm{ReO}_{3} \mathrm{~F}$ is more stable than even tet- $\mathrm{FReO}_{3}$, it is difficult to know exactly what phase the F-rich shell seen in EELS is composed of.
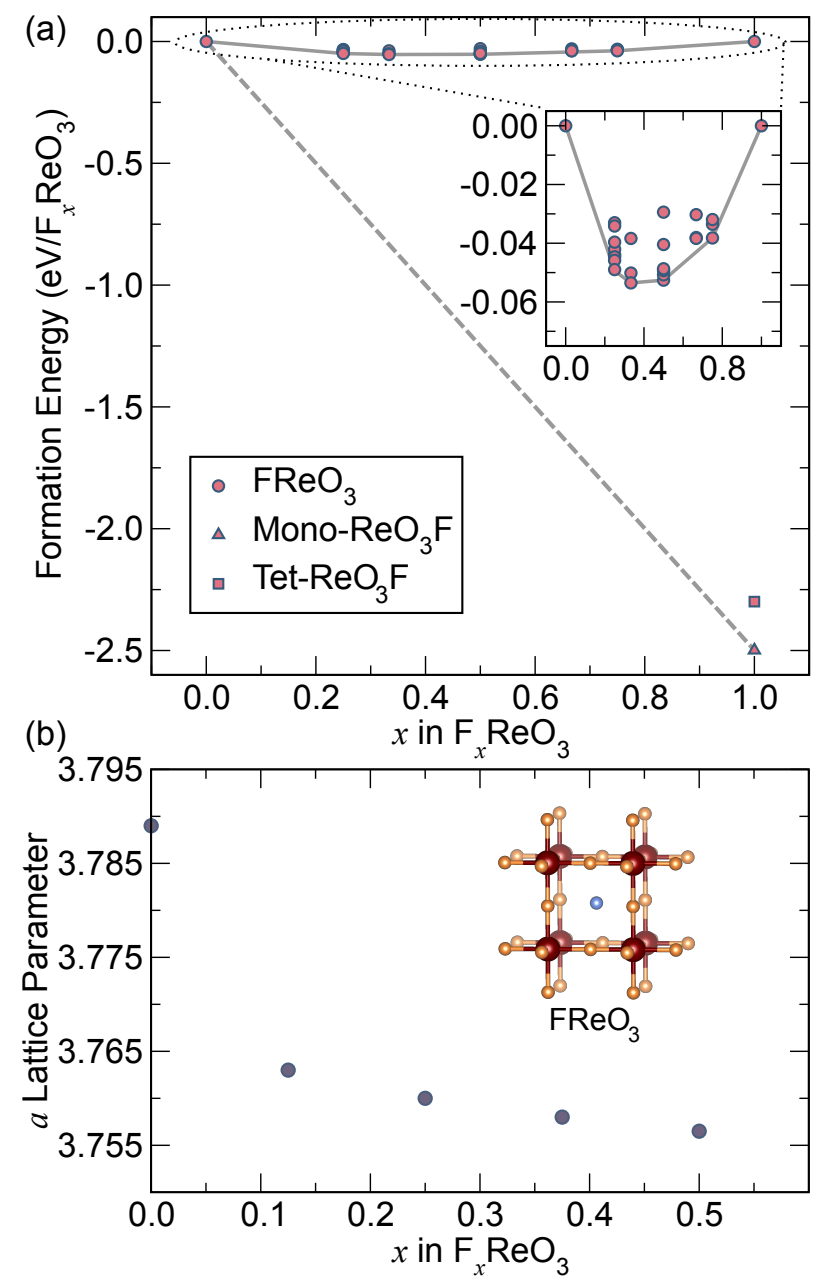

Figure 5: (a): The computed Hull diagram indicates that tetrahedral Re is energetically favorable upon fluoride intercalation while the inset shows a small energetic minima for the insertion of $\mathrm{F}$ to the A-site. (b): The calculated $a$ lattice parameter for $\mathrm{FReO}_{3}$ indicates a unit cell contraction, in agreement with operando XRD measurements.

For this, we turned to structural tools that were more sensitive to the local environments within the material such as neutron total scattering to generate Pair Distribution Function (PDF). Starting with the top panel of S.I. 
Figure S17, the ex situ neutron total scattering experiments for the pristine $\mathrm{ReO}_{3}$ are clearly well-described by the long-range average cubic structure whereas the significantly changed scattering from a washed sample of $\mathrm{F}_{0.6} \mathrm{ReO}_{3}$ is shown in the bottom portion of the same figure. The first two peaks at $1.88 \AA$ and $2.67 \AA$ in the pristine phase can be assigned to the $\mathrm{Re}-\mathrm{O}$ and $\mathrm{O}-\mathrm{O}$ nearest neighbors, which after oxidation, evolve into a complex mixture of peaks that makes precisely modeling the pattern exceptionally challenging. To aid in deconvoluting the contributions at each distance, the bottom panel of S.I. Figure S17 shows the calculated PDF adapted from the tet-FReO ${ }_{3}$ model considered earlier, as described in the Neutron Pair Distribution Analysis section of the Supporting Information. For example, the first shell of distances at $1.88 \AA$ splits into two distinct contributions that can be attributed to three short Re-O distances and one slightly longer Re-F bond within the newly created tetrahedra in the model. Furthermore, the peak at $3.75 \AA$, associated with Re-Re distances is seen to split as Re migrates from its starting position and the $\mathrm{O}-\mathrm{O}$ peak $(2.67 \AA)$ evolves into multiple peaks as new $\mathrm{O}-\mathrm{O}$ and $\mathrm{O}-\mathrm{F}$ environments are created. We also note that contributions from short $\mathrm{C}-\mathrm{H}$ and $\mathrm{C}-\mathrm{C}$ distance had to be included in order to account for all of the changes in the oxidized sample. These organic species are believed to be associated with a decomposition product created during the electrolyte decomposition at the counter electrode discussed earlier.

While the new distances in the PDF might initially appear to support the migration of Re into the tetrahedral interstitials, the predicted lattice parameters for such a hop suggest the unit cell should expand during such a transformation. In contrast, the operando diffraction data showed a contraction of the lattice, which would seem to imply that if such a hop were to occur it would need to be in an uncorrelated fashion that exhibits no long-range periodic order to the new tetrahedral sites. Alternatively, this may also suggest that a Re environment resembling the tetrahedral or seven coordinate models described previously might exist at the surface of the particles rather than as a completely separate new phase (see S.I. Figures S18-S21).

To clarify the ambiguity of the PDF data, solid-state ${ }^{19}$ F MAS NMR was used as a complementary probe of the local composition and structure of the intercalated fluoride ions. While the PDF results yield insights on locallyaveraged structural distortions within the bulk material, ${ }^{19} \mathrm{~F}$ MAS NMR allows for the direct detection and resolution of signals from fluoride species in different local environments. As shown in Figure S10 of the Supporting Information, the $1 \mathrm{D}{ }^{19} \mathrm{~F}$ MAS NMR spectrum for bulk $\mathrm{F}_{0.2} \mathrm{ReO}_{3}$, produced as a result of oxidative insertion during partial charge, exhibits a complicated distribution of ${ }^{19} \mathrm{~F}$ intensity over the frequency range of -100 to $-200 \mathrm{ppm}$. Interestingly, several distinct and relatively narrow ( $\sim 5 \mathrm{ppm}$, full-width-half-maximum) ${ }^{19} \mathrm{~F}$ signals are observed at isotropic chemical shifts of $-136,-141,-150,-170,-174$, and -189 ppm, which manifest fluorine species in well-defined local environments. The signal at $-136 \mathrm{ppm}$ is furthermore associated with a ${ }^{19} \mathrm{~F}$ spin-lattice relaxation time, $T_{1}$, of $4.0 \mathrm{~s}$ (Table T3, Supporting Information), which is consistent with fluoride in a diamagnetic perovskite environment. ${ }^{40,41}$ Interestingly, all of the other signals correspond to much shorter ${ }^{19} \mathrm{~F} T_{1}$ values of $0.3-0.4 \mathrm{~s}$ (Table T3), consistent with fluoride environments that are influenced by conducting or donor electrons. ${ }^{40-42}$ DFT calculations of several structural models selected from the phase diagram for fluoride intercalation into $\mathrm{ReO}_{3}$ predict an isotropic ${ }^{19} \mathrm{~F}$ 
chemical shift of -141 ppm for mono- $\mathrm{ReO}_{3} \mathrm{~F}$, close to the values of several of the measured signals. By comparison, the DFT calculations predict the isotropic chemical shift of a model structure with 7 -coordinate distorted-octahedra to be near $-73 \mathrm{ppm}$ and of tet- $\mathrm{FReO}_{3}$ to be near $-11 \mathrm{ppm}$, neither of which are experimentally observed (Figure S10). The multiple signals clearly show that the fluoride species in the electrode are inhomogeneously distributed in diverse local environments and corroborate the challenges to the analyses of the PDF data.

In a final attempt to reconcile the experimental results with computational models, X-ray spectroscopy was performed on the oxygen and fluorine K-edges for pristine and oxidized samples and compared with simulation based on the various structural permutations discussed previously. The O K-edge emission and absorption spectra reflect the bulk occupied and unoccupied O 2p partial density of states near the Fermi level. The oxygen K-edge, shown in Figure 6(c), evolves significantly after cycling, which clearly indicates a change in the electronic structure of $\mathrm{ReO}_{3}$ during oxidation. While the pristine material (blue) shows a large absorption peak at 0-2 eV associated with O $2 \mathrm{p}$ - Re $5 \mathrm{~d}$ bonding orbitals, these states are suppressed upon charging (pink), reflecting electron density being extracted from the material. The emergence of new peak at $9 \mathrm{eV}$ indicates a change to the local oxygen environment during oxidation. The presence of $\mathrm{F}^{-}$near very electrophilic $\mathrm{Re}^{6+}$ is expected to draw electron density from the O $2 p$ states to higher binding energies.

(a)
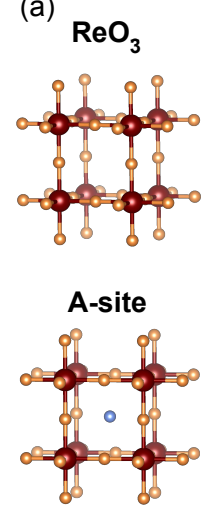
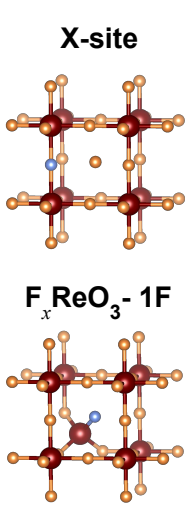

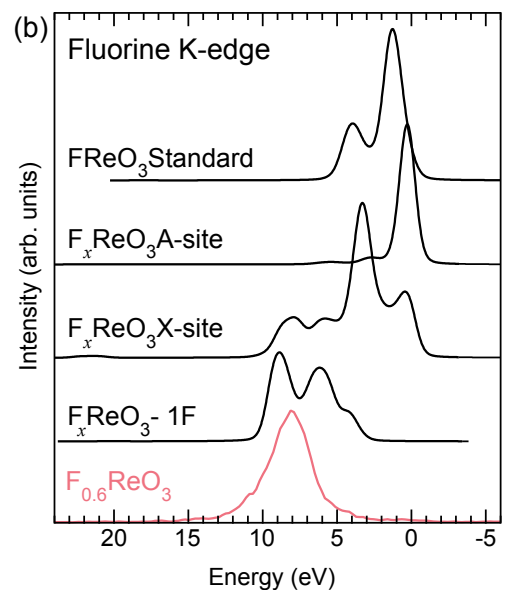

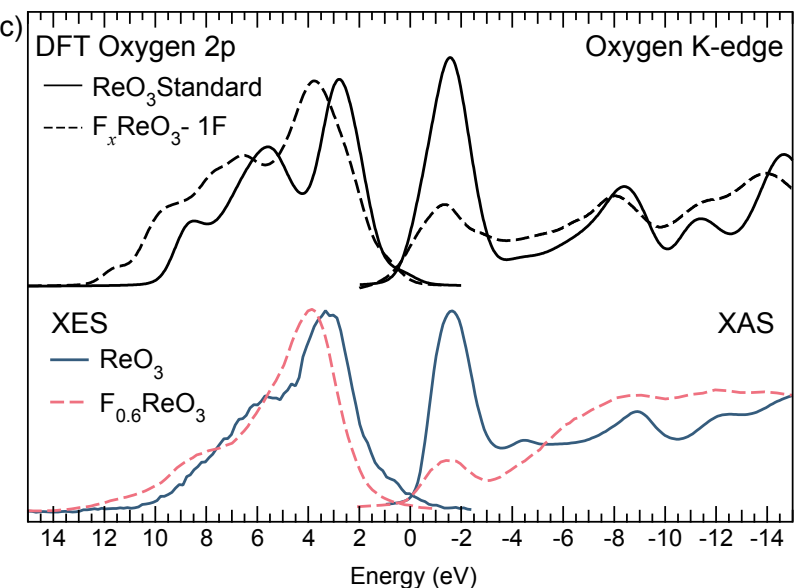

Figure 6: (a): Density functional theory calculations of the $\mathrm{O}$ and $\mathrm{F}$ K-edges were performed for different structural models of $\mathrm{FReO}_{3}$. (b) calculated X-ray emission spectra of the $\mathrm{F}$ K-edge are shown in black while the measured spectra of $\mathrm{ReO}_{3}$ is shown in orange and $\mathrm{F}_{0.6} \mathrm{ReO}_{3}$ is shown in blue. The energy shift of the measured spectra agrees well with the model of Re migration to the tetrahedral site. (c) The calculated and measured O K-edge X-ray emission and absorption spectra agree for both pristine and fluorinated $\mathrm{ReO}_{3}$ using the proposed structural model.

As shown in Figure 6(b) and (c), DFT calculations were used to model the expected O K-edge absorption and emission in both $\mathrm{ReO}_{3}$ and $\mathrm{FReO}_{3}$, using the structural models previously described, where Re migrates to one of the tetrahedral sites in $\mathrm{FReO}_{3}$. Additional models are provided in S.I. Figures S12 - S15. Curiously, the only model that captures the shape and energy shifts of the measured $\mathrm{O} 2 \mathrm{p}$ partial density of states is where a small number tetrahedral sites are created, rather than the phase that was predicted to be more thermodynamically favored i.e. where all of the Re jump to the tetrahedra (see S.I. Figure S15) The relative change in intensity between X-ray 
absorption peaks at $-2 \mathrm{eV}$ and $-9 \mathrm{eV}$, representing a change in $\mathrm{O}$ bonding environments as $\mathrm{ReO}_{3}$ is fluorinated, is matched by the DFT calculations, as is the shape and energy shift of the main emission features observed. The fluorescent nature of O K-edge XES, suggests that the spectral changes from the electrochemical reaction must not be limited to a surface reaction, but instead some change to the bulk $\mathrm{ReO}_{3}$ electronic structure must also occur. A closer look at the geometrically relaxed model containing a small number of tetrahedral Re shows a slight off-centering within the octahedral cages and some rotational disorder that likely explains the changes seen in the spectra.

Calculations of the fluorine K-edge for each model are shown as black traces in Figure 6(b). As seen, the measured XES spectra of $\mathrm{F}_{0.6} \mathrm{ReO}_{3}$ also agrees well with the model where Re migrates to stabilize the intercalated F-ions. While challenges with alignment of the F K-edge led to broadening of the measured signal and prevented distinct peaks from being resolved, the strong agreement between the calculated and measured energy shift further supports the presence of some tetrahedral species within the oxidized electrode.

Finally, HAXPES measurements were performed on the Re 4f, O 2p, and F 1s orbitals of pristine and fluorinated $\mathrm{ReO}_{3}$ and used to track changes in oxidation state of the various species during oxidative charging. As mentioned, the highly covalent bonding of $\mathrm{ReO}_{3}$ delocalizes electrons over both oxygen and Re states, which leads to partial changes in the Re oxidation state during charging. This can be seen in S.I. Figure S16, where the Re $4 \mathrm{f}$ photoemission was measured during various states of charge. The doublet peak at 43 and $46 \mathrm{eV}$ are diagnostic of Re ${ }^{6+}$, while a similar doublet at 46 and $48 \mathrm{eV}$ is attributed to $\mathrm{Re}^{7+}$. During oxidative charging, the formation of a shoulder at $48 \mathrm{eV}$ is observed as Re is partially oxidized. However, owing to the strong covalency, no evidence for a fully ionic description of $\operatorname{Re}^{7+}$ species was detected.

Taking all of the experimental and computational results together, there is clear evidence that $\mathrm{F}^{-}$ions have incorporated into $\mathrm{ReO}_{3}$ during oxidative charging, but the precise mechanism remains ambiguous. Operando Xray diffraction data shows a clear lattice contraction that agrees closely with the trends predicted for the models containing intercalated F-ions on the A-site of the $\mathrm{ReO}_{3}$ cage. Yet, this A-site model is predicted to be unstable compared to less densely packed polymorphs, and the ex situ materials characterization suggests the fluoride in the oxidized materials is most likely associated with those more stable tetrahedral tet-ReO ${ }_{3} \mathrm{~F}$ species or a decomposition to the structurally distinct mono- $\mathrm{ReO}_{3} \mathrm{~F}$ phase. This leads us to conclude that this discrepancy is likely due to changes in the material once it is removed from the electrochemical cell. Note that the operando diffraction experiments are collected from start to finish in approximately five hours with each pattern being collected in a matter of seconds. Hence, the most likely explanation for the discrepancy between the various characterization tools is that F-ions intercalate onto the A-site of the perovskite host, but quickly begin to decompose to the more thermodynamically favored phases over time.

It is curious that best agreement between the experimental data and the computation is found for the model containing a dilute number of Re that have jumped to tetrahedral sites. We suggest two possibilities to explain this observation. Either a shell of tetrahedrally coordinated Re forms as a decomposition product on the surface of the 
particles and the model we have considered is effectively capturing important interfacial effects; or, the correlated migration of Re into the tetrahedra environments is kinetically challenging and eventually results in some of the tetrahedral species trapped within the octahedral network even though they are thermodynamically disfavored. It is also possible that the final decomposition product contains some aspects of the dilute tetrahedral Re model, but the model fails to precisely capture all of the structural nuances. While we have gone to great lengths to evaluate as many possible forms of the intercalated structure, it is very likely that there are a vast number of alternate ways the perovskite lattice may be able to distort through octahedral tilting or cation migration. We therefore believe there is ample evidence to conclude that F-ions have incorporated into $\mathrm{ReO}_{3}$ during oxidation despite being unable to definitively assign a unique structural model to the resulting material. Further exploration using the thermodynamic modeling with a greatly expanded phase space along with additional operando local probes would be helpful in this regard, but falls outside the scope of the present study.

While the intercalation of fluoride ions into structures as dense as perovskite may initially seems too energetically demanding, several studies have shown the possibility to intercalate fluoride perovskite derivatives using aggressive chemical oxidants. ${ }^{43-46}$ Other work has investigated the formation of interstitial fluoride species within similar $\mathrm{Sr}_{3} \mathrm{Ru}_{2} \mathrm{O}_{7}$ species, in which layers of fluoride are formed and result in correlated rotations of the $\mathrm{RuO}_{6}$ octahedra. ${ }^{47}$ Crucially, fluorination of the parent oxide was performed at a low reaction temperature of 220 to $300^{\circ} \mathrm{C}$, demonstrating the relative ease of fluoride intercalation into an oxide.

It is also known that halide ions are very mobile within the perovskite structures, with studies of $\mathrm{CH}_{3} \mathrm{NH}_{3} \mathrm{PbI}_{3}$ showing this migration occurs easily under an applied bias. ${ }^{48,49}$ These reactions are typically limited by halide diffusion through the structure via interstitials or vacancies but can proceed quite quickly. In fact, Walsh and Stranks have clearly shown that both the cationic and anionic sublattices in perovksites are mobile and the activation barrier for defect migration is relatively low, which is in good agreement with what we observe here. ${ }^{50}$

In summary, we have presented, for the first time, the oxidative fluorination of the dense oxide $\mathrm{ReO}_{3}$ using electrochemical cells and a liquid electrolyte at room temperature. We find that electrons are removed from the strongly hybridized Re-O orbitals at the Fermi level resulting in partial oxidation of both Re and O, with charge compensation occurring via fluoride intercalation into the vacant perovskite $A$-site. The intercalation of fluoride results in complex structural changes as some Re appear to migrates from the octahedral sites to newly formed tetrahedral sites, as evidenced by changes in the XAS and XES spectra, SXRD patterns, and neutron PDF scattering. While DFT calculations clearly shows that the intercalated form of $\mathrm{FReO}_{3}$ is strongly disfavored by thermodynamics, more exhaustive explorations of the phase space are necessary to fully understand whether more complex tilting/alterations of the initial octahedral network may service to stabilize these phases. Fluoride intercalation at ambient conditions represents a paradigm shift compared to traditional Li-ion battery technologies and offers a window into a broad new avenue for developing methods for higher storage capacity if cationic and anionic intercalation can be successfully and sequentially achieved within single phase materials. 
Supporting Information The Supporting Information is available free of charge.

- Experimental Methods, including Materials Synthesis, Materials Characterization, Electrochemical Characterization, Differential Electrochemical Mass Spectrometry, Solid-State ${ }^{19}$ F NMR Spectroscopy, and Computational Methods; additional operando X-ray diffraction measurements and simulations; impedance spectroscopy measurements; solid state NMR calculations and measurements; neutron PDF measurements

Author Information The authors declare no competing financial interest.

Acknowledgments N.H.B, A.I., S.M.B, J.V., E.T.M., B.F.C, A.V.d.V. S.R.N. and B.C.M. were supported as part of the Center for Synthetic Control Across Length-scales for Advancing Rechargeables (SCALAR), an Energy Frontier Research Center funded by the U.S. Department of Energy, Office of Science, Basic Energy Sciences under Award No DE-SC0019381. We acknowledge support through the Research Corporation for Science Advancement (RSCA) for a Scialog grant. L.F.J.P. also gratefully acknowledges support from the RSCA through an Advanced Energy Storage Scialog award. W.R. and D.O.S. acknowledge funding by the Faraday Institution (http://www.faraday.ac.uk, EP/S003053/1, grant no. FIRG003) and the use of the MICHAEL computing cluster. D.O.S. acknowledges support from the European Research Council, ERC, (Grant 758345). Via our membership of the UK's HEC Materials Chemistry Consortium, which is funded by the EPSRC (EP/L000202, EP/R029431), this work used the ARCHER UK National Supercomputing Service (http://www.archer.ac.uk). Use of the Advanced Photon Source at Argonne National Laboratory was supported by the U.S. Department of Energy, Office of Science, Office of Basic Energy Sciences, under Contract No. DE-AC02-06CH11357. This research used resources of the Advanced Light Source and the Molecular Foundry which are U.S. DOE Office of Science facilities at Lawrence Berkeley National Laboratory under Contract No. DE-AC02-05CH11231. We acknowledge Diamond Light Source for time on Beamline I09 under Proposal SI22250. The neutron pair distribution function measurements used resources at Spallation Neutron Source, a DOE Office of Science User Facility operated by the Oak Ridge National Laboratory. Materials characterization made use of the MRL Shared Experimental Facilities at UCSB, which are supported by the MRSEC Program of the NSF under Award No. DMR 1720256; a member of the NSF-funded Materials Research Facilities Network. The content of the information does not necessarily reflect the position or the policy of the U. S. Government, and no official endorsement should be inferred. We thank Matthew Mecklenburg for his assistance with TEM and EELS measurements at the University of Southern California Core Center of Excellence in Nano Imaging. We acknowledge and thank Prof. Clare Grey for early discussions and the use of her NMR spectrometers. We thank Sara Abdel Razek for assistance with the x-ray absorption/emission simulations.

\section{References}

(1) Whittingham, M. S. Lithium Batteries and Cathode Materials. Chem. Rev. 2004, 104, 4271-4302. 
(2) Mortimer, R. J. Electrochromic materials. Chem. Soc. Rev. 1997, 26, 147.

(3) Rives, V.; del Arco, M.; Martín, C. Intercalation of drugs in layered double hydroxides and their controlled release: A review. Appl. Clay Sci. 2014, 88-89, 239-269.

(4) Manthiram, A. A reflection on lithium-ion battery cathode chemistry. Nat. Commun. 2020, 11, 1550.

(5) Nazri, G.; Pistoia, G. Lithium Batteries: Science and Technology; Springer US, 2008.

(6) Gamble, F. R.; Osiecki, J. H.; Cais, M.; Pisharody, R.; DiSalvo, F. J.; Geballe, T. H. Intercalation Complexes of Lewis Bases and Layered Sulfides: A Large Class of New Superconductors. Science 1971, 174, 493-497.

(7) Whittingham, M. S.; Jacobson, A. J. Intercalation chemistry; Academic Press, 1982.

(8) West, W. C.; Whitacre, J. F.; Leifer, N.; Greenbaum, S.; Smart, M.; Bugga, R.; Blanco, M.; Narayanan, S. R. Reversible Intercalation of Fluoride-Anion Receptor Complexes in Graphite. J. Electrochem. Soc. 2007, 154, A929.

(9) Schafhaeutl, C. Ueber die Verbindungen des Kohlenstoffes mit Silicium, Eisen und anderen Metallen, welche die verschiedenen Gallungen von Roheisen, Stahl und Schmiedeeisen bilden. J. Prakt. Chem. 1840, 21, $129-157$.

(10) Rüdorff, W.; Hofmann, U. Über Graphitsalze. Z. Anorg. Allg. Chem. 1938, 238, 1-50.

(11) Beck, F.; Junge, H.; Krohn, H. Graphite intercalation compounds as positive electrodes in galvanic cells. Electrochim. Acta 1981, 26, 799-809.

(12) Beltrop, K.; Meister, P.; Klein, S.; Heckmann, A.; Grünebaum, M.; Wiemhöfer, H.-D.; Winter, M.; Placke, T. Does Size really Matter? New Insights into the Intercalation Behavior of Anions into a Graphite-Based Positive Electrode for Dual-Ion Batteries. Electrochim. Acta 2016, 209, 44-55.

(13) Mallouk, T.; Bartlett, N. Reversible intercalation of graphite by fluorine: a new bifluoride, $\mathrm{C}_{12} \mathrm{HF}_{2}$, and graphite fluorides, $\mathrm{C}_{x} \mathrm{~F}(5>\mathrm{x}>2)$. J. Chem. Soc., Chem. Commun. 1983, 103.

(14) Carlin, R. T. Dual Intercalating Molten Electrolyte Batteries. J. Electrochem. Soc. 1994, 141, L73.

(15) Deunf, É.; Jiménez, P.; Guyomard, D.; Dolhem, F.; Poizot, P. A dual-ion battery using diamino-rubicene as anion-inserting positive electrode material. Electrochem. Commun. 2016, 72, 64-68.

(16) Seel, J. A.; Dahn, J. R. Electrochemical Intercalation of $\mathrm{PF}_{6}$ into Graphite. J. Electrochem. Soc. 2000, 147, 892. 
(17) Schoonman, J. A Solid-State Galvanic Cell with Fluoride-Conducting Electrolytes. J. Electrochem. Soc. 1976, 123, $1772-1775$.

(18) Gschwind, F.; Rodriguez-Garcia, G.; Sandbeck, D. J. S.; Gross, A.; Weil, M.; Fichtner, M.; Hörmann, N. Fluoride ion batteries: Theoretical performance, safety, toxicity, and a combinatorial screening of new electrodes. J. Fluorine Chem. 2016, 182, 76-90.

(19) Grenier, A.; Porras-Gutierrez, A.-G.; Groult, H.; Beyer, K. A.; Borkiewicz, O. J.; Chapman, K. W.; Dambournet, D. Electrochemical reactions in fluoride-ion batteries: mechanistic insights from pair distribution function analysis. J. Mater. Chem. A 2017, 5, 15700-15705.

(20) Schoonman, J.; Oversluizen, G.; Wapenaar, K. Solid electrolyte properties of LaF 3 . Solid State Ion. 1980, 1, $211-221$.

(21) Roos, A.; Aalders, A.; Schoonman, J.; Arts, A.; de Wijn, H. Electrical conduction and ${ }^{19}$ F NMR of solid solutions $\mathrm{La}_{1-x} \mathrm{Ba}_{x} \mathrm{~F}_{3-x}$. Solid State Ion. 1983, 9-10, 571-574.

(22) Breuer, S.; Gombotz, M.; Pregartner, V.; Hanzu, I.; Wilkening, M. Heterogeneous F anion transport, local dynamics and electrochemical stability of nanocrystalline $\mathrm{La}_{1-x} \mathrm{BaF}_{3}$. Energy Storage Mater. 2019, 16, 481490.

(23) A. Reddy, M.; Fichtner, M. Batteries based on fluoride shuttle. J. Mater. Chem. 2011, $21,17059$.

(24) Rongeat, C.; Reddy, M. A.; Diemant, T.; Behm, R. J.; Fichtner, M. Development of new anode composite materials for fluoride ion batteries. J. Mater. Chem. A 2014, 2, 20861-20872.

(25) Thieu, D. T.; Fawey, M. H.; Bhatia, H.; Diemant, T.; Chakravadhanula, V. S. K.; Behm, R. J.; Kübel, C.; Fichtner, M. $\mathrm{CuF}_{2}$ as Reversible Cathode for Fluoride Ion Batteries. Adv. Funct. Mater. $2017,27,1701051$.

(26) Nowroozi, M. A.; Ivlev, S.; Rohrer, J.; Clemens, O. $\mathrm{La}_{2} \mathrm{CoO}_{4}$ : a new intercalation based cathode material for fluoride ion batteries with improved cycling stability. J. Mater. Chem. A 6, 4658-4669.

(27) Nowroozi, M.; Clemens, O. Insights on the Behavior of Conversion-Based Anode Materials for Fluoride Ion Batteries by Testing against an Intercalation-Based Reference Cathode. ACS Appl. Energy Mater. 2018, 1, $6626-6637$.

(28) Zaheer, W.; Andrews, J. L.; Parija, A.; Hyler, F. P.; Jaye, C.; Weiland, C.; Yu, Y.-S.; Shapiro, D. A.; Fischer, D. A.; Guo, J.; Velázquez, J. M.; Banerjee, S. Reversible Room-Temperature Fluoride-Ion Insertion in a Tunnel-Structured Transition Metal Oxide Host. ACS Energy Lett. 2020, 2520-2526. 
(29) Christe, K. O.; Wilson, W. W.; Wilson, R. D.; Bau, R.; Feng, J. A. Syntheses, properties, and structures of anhydrous tetramethylammonium fluoride and its 1:1 adduct with trans-3-amino-2-butenenitrile. J. Am. Chem. Soc. 1990, 112, 7619-7625.

(30) Sharma, R. K.; Fry, J. L. Instability of anhydrous tetra-n-alkylammonium fluorides. J. Org. Chem. 1983, 48, $2112-2114$.

(31) Davis, V. K. et al. Room-temperature cycling of metal fluoride electrodes: Liquid electrolytes for high-energy fluoride ion cells. Science 2018, 362, 1144-1148.

(32) Bashian, N. H.; Zhou, S.; Zuba, M.; Ganose, A. M.; Stiles, J. W.; Ee, A.; Ashby, D. S.; Scanlon, D. O.; Piper, L. F. J.; Dunn, B.; Melot, B. C. Correlated Polyhedral Rotations in the Absence of Polarons during Electrochemical Insertion of Lithium in $\mathrm{ReO}_{3}$. ACS Energy Lett. 2018, 3, 2513-2519.

(33) Meisel, K. Rheniumtrioxyd. III. Mitteilung. Über die Kristallstruktur des Rheniumtrioxyds. Z. Anorg. Allg. Chem. 1932, 207, 121-128.

(34) Ferretti, A.; Rogers, D. B.; Goodenough, J. B. The relation of the electrical conductivity in single crystals of rhenium trioxide to the conductivities of $\mathrm{Sr}_{2} \mathrm{MgReO}_{6}$ and $\mathrm{Na}_{x} \mathrm{WO}_{3}$. J. Phys. Chem. Solids 1965, 26, 2007-2011.

(35) Tran, N.; Croguennec, L.; Menetrier, M.; Weill, F.; Biensan, P.; Jordy, C.; Delmas, C. Mechanisms Associated with the "Plateau" Observed at High Voltage for the Overlithiated $\mathrm{Li}_{1.12}\left(\mathrm{Ni}_{0.425} \mathrm{Mn}_{0.425} \mathrm{Co}_{0.15}\right)_{0.88} \mathrm{O}_{2}$ System. Chem. Mater. 2008, 20, 4815-4825.

(36) Armstrong, A. R.; Holzapfel, M.; Novák, P.; Johnson, C. S.; Kang, S.-H.; Thackeray, M. M.; Bruce, P. G. Demonstrating Oxygen Loss and Associated Structural Reorganization in the Lithium Battery Cathode $\mathrm{Li}\left[\mathrm{Ni}_{0.2} \mathrm{Li}_{0.2} \mathrm{Mn}_{0.6}\right] \mathrm{O}_{2}$. J. Am. Chem. Soc. 2006, 128, 8694-8698.

(37) Van der Ven, A.; Deng, Z.; Banerjee, S.; Ong, S. P. Rechargeable Alkali-Ion Battery Materials: Theory and Computation. Chem. Rev. 2020, 120, 6977-7019.

(38) Zagorac, D.; Müller, H.; Ruehl, S.; Zagorac, J.; Rehme, S. Recent developments in the Inorganic Crystal Structure Database: theoretical crystal structure data and related features. J. Appl. Crystallogr. 2019, 52.

(39) Supeł, J.; Marx, R.; Seppelt, K. Preparation and Structure of Rhenium Fluoride Trioxide $\mathrm{ReO}_{3} \mathrm{~F}$, and the Polymorphism of Rhenium Trifluoride Dioxide, $\mathrm{ReO}_{2} \mathrm{~F}_{3}$. Z. Anorg. Allg. Chem. 2005, 631, 2979-2986.

(40) Ruiz-Preciado, M. A.; Kubicki, D. J.; Hofstetter, A.; McGovern, L.; Futscher, M. H.; Ummadisingu, A.; Gershoni-Poranne, R.; Zakeeruddin, S. M.; Ehrler, B.; Emsley, L.; Milić, J. V.; Grätzel, M. Supramolecular Modulation of Hybrid Perovskite Solar Cells via Bifunctional Halogen Bonding Revealed by Two-Dimensional ${ }^{19}$ F Solid-State NMR Spectroscopy. J. Am. Chem. Soc. 2020, 142, 1645-1654. 
(41) Cho, S. H.; Ghosh, S.; Berkson, Z. J.; Hachtel, J. A.; Shi, J.; Zhao, X.; Reimnitz, L. C.; Dahlman, C. J.; Ho, Y.; Yang, A.; Liu, Y.; Idrobo, J.-C.; Chmelka, B. F.; Milliron, D. J. Syntheses of Colloidal F:In ${ }_{2} \mathrm{O}_{3}$ Cubes: Fluorine-Induced Faceting and Infrared Plasmonic Response. Chem. Mater. 2019, 31, 2661-2676.

(42) Yesinowski, J. P.; Berkson, Z. J.; Cadars, S.; Purdy, A. P.; Chmelka, B. F. Spatially correlated distributions of local metallic properties in bulk and nanocrystalline GaN. Phys. Rev. B 2017, 95.

(43) Greaves, C.; Francesconi, M. G. Fluorine insertion in inorganic materials. Curr. Opin. Solid State Mater. Sci. 1998, 3, 132-136.

(44) Greaves, C.; Kissick, J. L.; Francesconi, M. G.; Aikens, L. D.; Gillie, L. J. Synthetic strategies for new inorganic oxide fluorides and oxide sulfates. J. Mater. Chem. 1999, 9, 111-116.

(45) McCabe, E. E.; Greaves, C. Fluorine insertion reactions into pre-formed metal oxides. J. Fluorine Chem. 2007, $128,448-458$.

(46) Case, G. S.; Hector, A. L.; Levason, W.; Needs, R. L.; Thomas, M. F.; Weller, M. T. Syntheses, powder neutron diffraction structures and Mössbauer studies of some complex iron oxyfluorides: $\mathrm{Sr}_{3} \mathrm{Fe}_{2} \mathrm{O}_{6} \mathrm{~F}_{0.87}, \mathrm{Sr}_{2} \mathrm{FeO}_{3} \mathrm{~F}$ and $\mathrm{Ba}_{2} \mathrm{InFeO}_{5} \mathrm{~F}_{0.68}$. J. Mater. Chem. 1999, 9, 2821-2827.

(47) Li, R. K.; Greaves, C. Double-layered ruthenate $\mathrm{Sr}_{3} \mathrm{Ru}_{2} \mathrm{O}_{7} \mathrm{~F}_{2}$ formed by fluorine insertion into $\mathrm{Sr}_{3} \mathrm{Ru}_{2} \mathrm{O}_{7} . P h y s$. Rev. B 2000, 62, 3811-3815.

(48) Pellet, N.; Teuscher, J.; Maier, J.; Grätzel, M. Transforming Hybrid Organic Inorganic Perovskites by Rapid Halide Exchange. Chem. Mater. 2015, 27, 2181-2188.

(49) Elmelund, T.; Scheidt, R. A.; Seger, B.; Kamat, P. V. Bidirectional Halide Ion Exchange in Paired Lead Halide Perovskite Films with Thermal Activation. ACS Energy Lett. 2019, 4, 1961-1969.

(50) Walsh, A.; Stranks, S. D. Taking Control of Ion Transport in Halide Perovskite Solar Cells. ACS Energy Lett. 2018, 3, 1983-1990. 